Revista Española de

Cirugía Oral y Maxilofacial

www.elsevier.es/recom

Caso clínico

\title{
Fascitis nodular cervical en paciente gestante: revisión de la literatura y presentación de un caso
}

\author{
Manuel Acosta-Feria ${ }^{a, *}$, Benito Ramos-Medina ${ }^{a}$, Maribel Sánchez-Zapata ${ }^{b}$, \\ Antonio Gómez-Poveda ${ }^{a}$, Juan José Haro Luna ${ }^{a}$, Raquel Villar Puchades ${ }^{a}$ \\ y María José Sánchez de las Matas Garrec \\ aServicio de Cirugía Oral y Maxilofacial, Hospital Santa María del Rosell, Cartagena, Murcia, España. \\ bServicio de Ginecología, Hospital Santa María del Rosell, Cartagena, Murcia, España. \\ 'Servicio de Anatomía Patológica, Hospital Santa María del Rosell, Cartagena, Murcia, España.
}

INFORMACIÓN DEL ARTÍCULO

Historia del artículo:

Recibido el 18 de abril de 2010

Aceptado el 21 de septiembre de

2010

Palabras clave:

Fascitis nodular;

Embarazo;

Cabeza y cuello;

Glándula parótida

Keywords:

Nodular fasciitis;

Pregnancy;

Head and neck;

Parotid gland

\section{R E S U M E N}

La fascitis nodular es una lesión benigna rara, de crecimiento rápido, cuya patogenia es aún desconocida. Si bien a nivel de cabeza y cuello aparece en un $20 \%$ de los casos, su aparición en pacientes gestantes es extremadamente rara. Presentamos el segundo caso descrito en la literatura en el cual está presente dicha asociación.

Mujer de 25 años y gestante de 13 semanas, con una tumoración retroauricular derecha de $3 \mathrm{~cm}$ de diámetro, 3 meses de evolución y rápido crecimiento. No presentaba parálisis facial ni dolor en la exploración inicial. Tras la exéresis quirúrgica de la lesión, el diagnóstico anatomopatológico definitivo de ésta, fue de fascitis nodular. Tras 2 años desde la intervención quirúrgica no se ha evidenciado recidiva de la tumoración, llevándose el embarazo a término sin presentar complicaciones ni para la madre ni para el feto.

(c) 2010 SECOM. Publicado por Elsevier España, S.L. Todos los derechos reservados.

Cervical nodular fasciitis in a pregnant woman: Review of the literature and presentation of a new case

A B S T R A C T

The nodular fasciitis is a rare benign lesion of rapid growth, whose pathogenesis is still unknown. While at the head and neck appeared in $20 \%$ of cases, its occurrence in pregnant patients is extremely rare. We report the second case described in the literature in which this association is present.

Woman of 25 years and 13 weeks pregnant, who had a right auricular tumour $3 \mathrm{~cm}$ in diameter, 3 months of development and rapid growth. No facial paralysis or pain in the initial exploration.

\footnotetext{
${ }^{*}$ Autor para correspondencia.

Correo electrónico: Te_Ba@hotmail.com (Manuel Acosta-Feria).
} 
After surgical resection of the lesion, the final pathological diagnosis of the same was nodular fasciitis. After two years since the surgery, there was no evidence of recurrence of the tumour, taking ad términun pregnancy without complications or the mother or the fetus.

○ 2010 SECOM. Published by Elsevier España, S.L. All rights reserved.

\section{Introducción}

La fascitis nodular, descrita por Konwaller et al en 1955, es también conocida como fascitis seudosarcomatosa, fibromatosis seudosarcomatosa, fascitis proliferativa o fascitis infiltrativa $^{1,2}$. Es una lesión benigna de los tejidos blandos, producida fundamentalmente por una proliferación de fibroblastos. Es más común en adultos jóvenes, y la localización más frecuente es en las extremidades superiores, seguida del tronco, y sólo entre el 7 y el $20 \%$ de los casos se origina en la cabeza y el cuello, apareciendo generalmente como una masa alargada y normalmente no ulcerada ${ }^{3-5}$. Las lesiones en la mayoría de los casos miden menos de $5 \mathrm{~cm}$, aunque en ocasiones pueden alcanzar más de $10 \mathrm{~cm}$. Crecen típicamente a nivel subcutáneo, aunque se han visto en ocasiones lesiones a nivel intramuscular o que afectan a órganos tales como la glándula parótida 5 .

La fascitis nodular se puede confundir histológica y clínicamente con tumoraciones malignas, por lo que es muy importante el diagnóstico diferencial a través del estudio anatomopatológico. El diagnóstico diferencial se ve dificultado en muchas ocasiones debido a la rareza de esta entidad clínica y su lugar de presentación. Además, el rápido crecimiento de la tumoración, su alta celularidad, la alta actividad mitótica y la posible infiltración de los bordes tumorales hacen que en ocasiones la lesión sea erróneamente diagnosticada como un tumor maligno de origen mesenquimatoso. Sin embargo, nunca presenta atipias celulares. Desde el punto de vista radiológico no existen hallazgos patognomónicos en las pruebas de imagen más utilizadas para su diagnóstico (tomografía computarizada [TC] o resonancia magnética $[\mathrm{RM}])^{1,3,6}$. En el $40 \%$ de los casos se ha relacionado la tumoración con la presencia de un traumatismo previo ${ }^{6,7}$.

Presentamos el segundo caso descrito en la literatura en el cual coinciden fascitis nodular junto con embarazo. Nos planteamos la siguiente pregunta: ¿existe relación entre ambos debido a alteraciones hormonales propias del estado gestacional?

\section{Caso clínico}

Mujer de 25 años de edad y gestante de 21 semanas que acude al servicio de cirugía oral y maxilofacial por presentar una tumoración a nivel retroauricular derecho de 3 meses de evolución y rápido crecimiento. A la exploración, la lesión era dura y móvil, de aproximadamente $5 \mathrm{~cm}$ de diámetro, dolorosa a la palpación, con eritema peritumoral. No presentaba afectación del nervio facial (fig. 1).

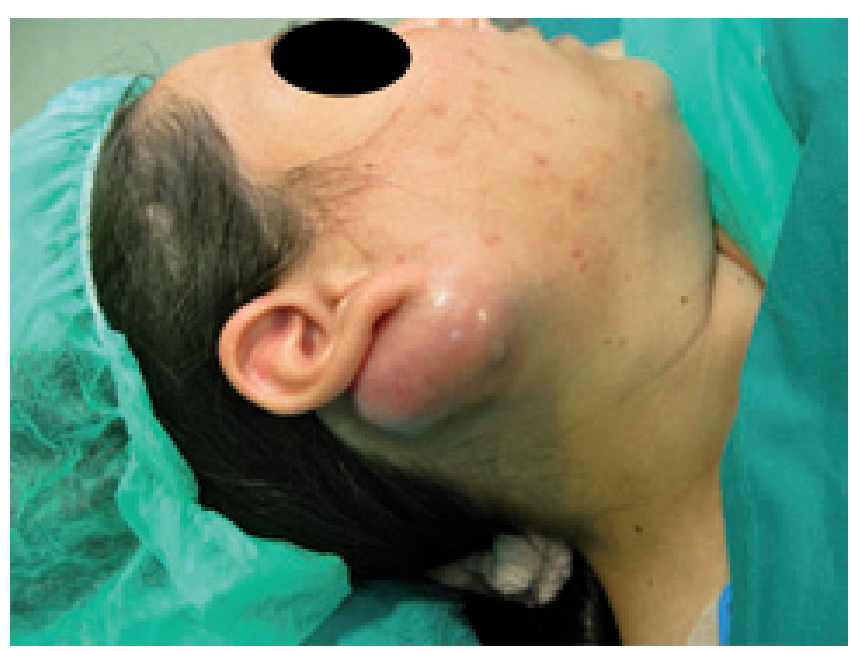

Figura 1 - Tumoración retroauricular.

Llevamos a cabo una nueva punción-aspiración con aguja fina (PAAF), la cual indica la presencia de una neoplasia mesenquimal de células epitelioides y fusiformes de aspecto fibrohistiocítico, y el anatomopatólogo nos aconseja la exéresis quirúrgica completa de la lesión, dado que no se pudo descartar el carácter de malignidad en la tumoración. Con los datos clínicos obtenidos, junto con la rápida evolución de la lesión, el informe del estudio anatomopatológico y la realización de una RM como prueba de imagen, y tras consultar con el servicio de ginecología y obstetricia de nuestro hospital, decidimos la extirpación de la tumoración, que se realizó al final del segundo trimestre de la gestación. Realizamos una incisión preauricular, con extensión cervical rodeando la tumoración, haciendo una disección por planos hasta localizar el tronco principal del nervio facial, al que pudimos respetar. Resecamos la lesión hasta el plano del esternocleidomastoideo, separándolo del mismo, y reconstruyendo por último la zona intervenida mediante injerto libre de piel hendida (fig. 2).

Tras la intervención quirúrgica, el embarazo trascurrió sin complicaciones, tanto para la madre como para el feto, sin que se observe afectación del nervio facial en la actualidad, ni recidiva de la lesión (fig. 3 A y B).

El diagnóstico anatomopatológico definitivo de la pieza quirúrgica nos informó que estábamos ante una neoplasia mesenquimal constituida por células fusiformes de hábito fibroblástico, sin pleomorfismo nuclear ni hipercromasia, con índice mitótico bajo. Se visualizan zonas celulares con patrón arremolinado, con frecuentes células multinucleadas del tipo osteoclástico. La neoplasia no infiltra la dermis, está circunscrita pero no encapsulada, e infiltra focalmente en la porción 


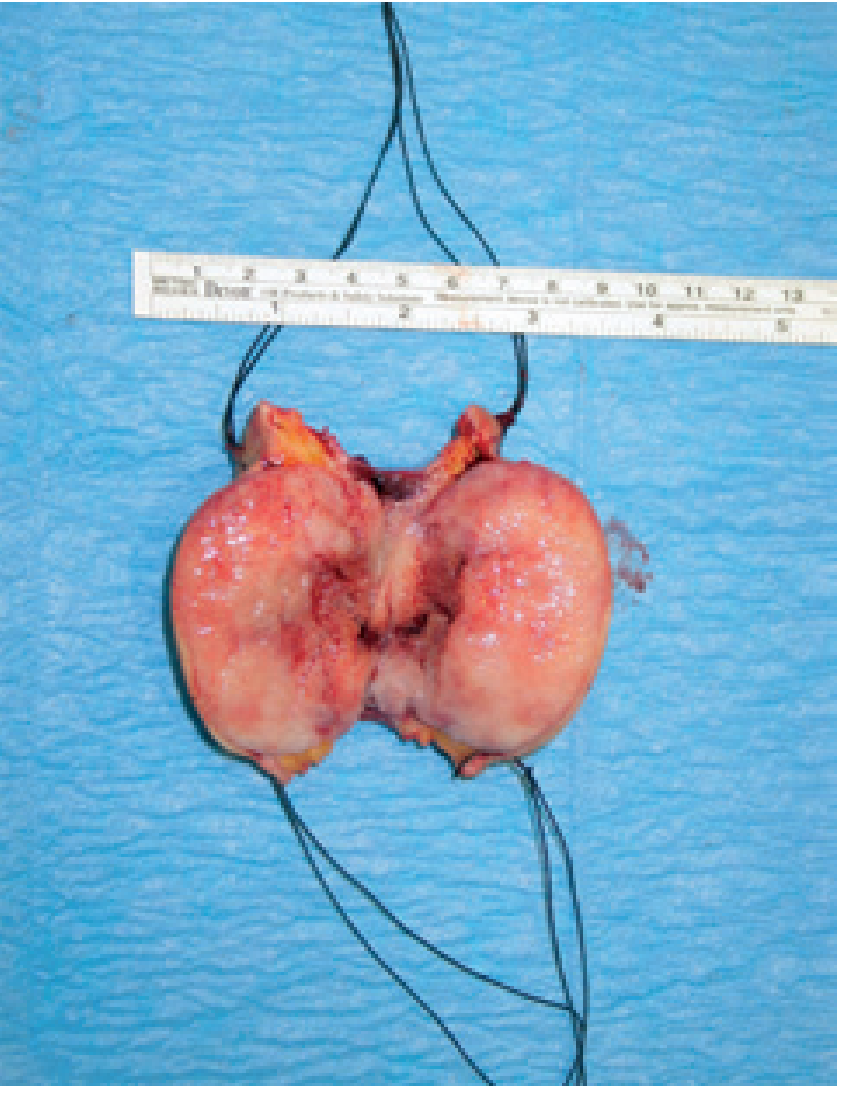

Figura 2 - Lesión extirpada, aspecto macroscópico. profunda, la glándula parótida. La lesión es diagnosticada como neoplasia mesenquimal fusocelular fibroblástica/miofibroblástica compatible con fascitis nodular (fig. 4 A-C).

\section{Discusión}

La fascitis nodular constituye una lesión benigna del tejido conectivo. Ha sido denominada también en la literatura como fascitis seudosarcomatosa, fascitis proliferativa y fibromatosis seudosarcomatosa, y en la actualidad es la lesión seudosarcomatosa más frecuente de los tejidos blandos ${ }^{8-11}$. Su importancia radica en el hecho de que ocasionalmente es diagnosticada erróneamente como un sarcoma, tanto desde el punto de vista clínico debido a su rápido crecimiento sin signos de infección, como histológico debido a su alta celularidad, actividad mitótica e infiltración de bordes ${ }^{12,13}$. Cuando se presenta en la región de cabeza y cuello, raramente lo hace en la mucosa oral, y su localización más frecuente es en la piel de la cara, la región parotídea y en el tejido subcutáneo de la mandíbula y en la región malar o zigoma 9 .

La presentación clínica más frecuente de la lesión consiste en una tumoración solitaria, de rápido crecimiento, asociada a dolor e inflamación. Menos frecuentemente puede asociarse a compresión de nervio periférico, produciendo parestesias. La aparición de lesiones múltiples es extremadamente rara. El tamaño menor de $5 \mathrm{~cm}$ es el más estudiado. Thompson $^{4}$ realizó un estudio retrospectivo de 50 casos de fascitis
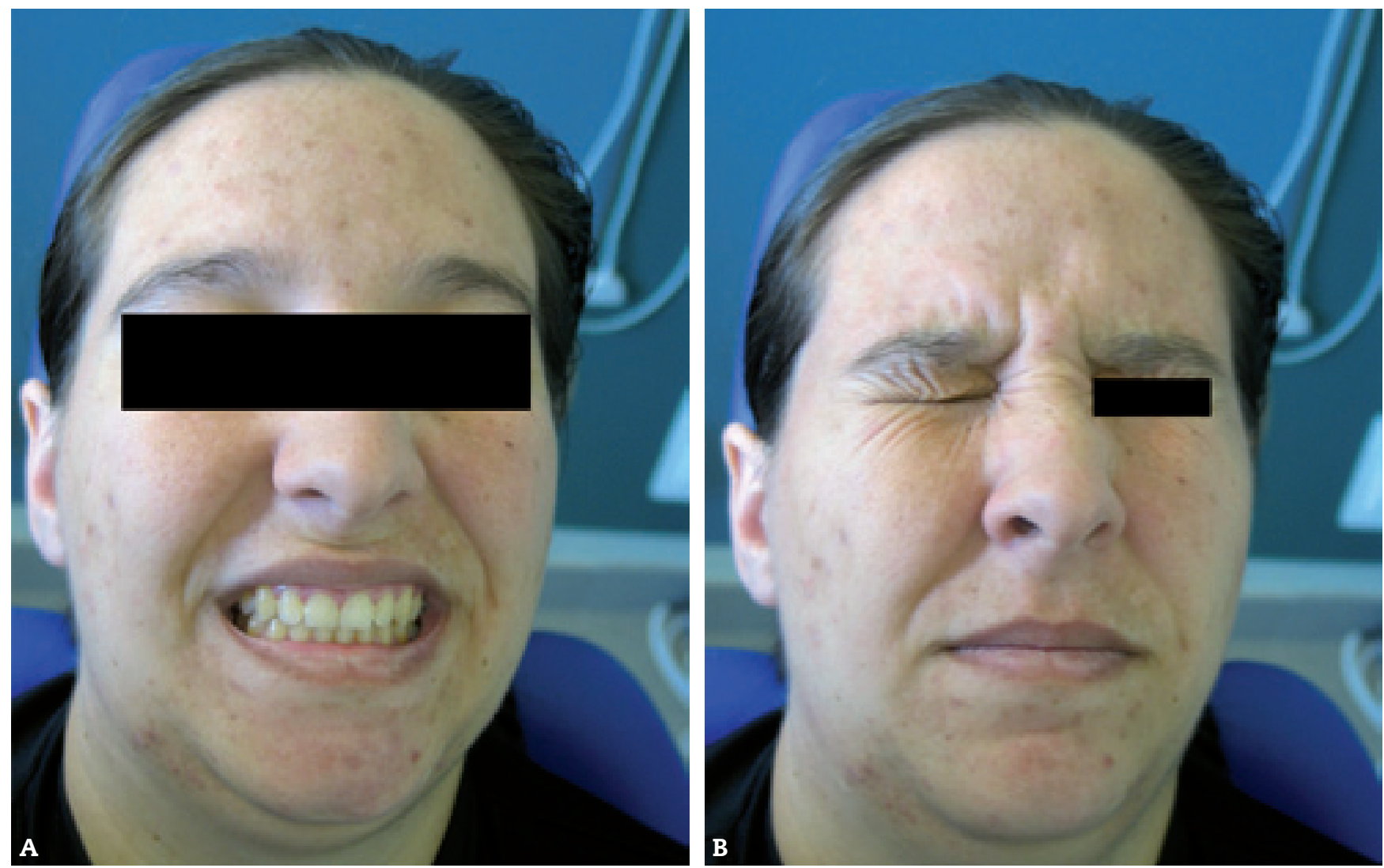

Figura 3 - A) Exploración de la rama marginal mandibular. B) Exploración de la rama cigomático-frontal. 

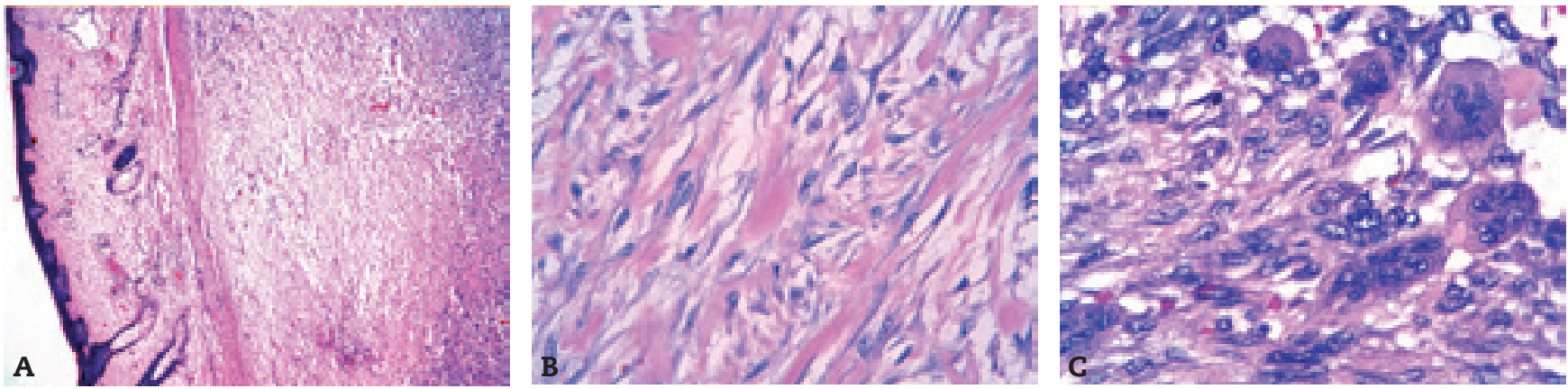

Figura 4 - A) En la imagen se aprecia a bajo aumento aplanamiento de la epidermis y subyacente neoplasia bien delimitada, nodular con células fusiformes que forman haces de aspecto arremolinado (tinción HE, $\times 40$ ). B) Imagen de células fusiformes de hábito fibroblástico con patrón de crecimiento arremolinado y haces de colágeno queloidal, fibras esquenoides (tinción HE, $\times 400$ ). C) Imagen de células fusiformes de hábito fibroblástico y células gigantes multinucleadas de hábito osteoclástico (tinción HE, $\times 630$ ).

nodular auricular, 22 mujeres y 28 hombres, con una edad media de 27,4 años. El $98 \%$ de los pacientes presentaban una masa tumoral, con un tamaño medio de 1,9 cm. Los síntomas más frecuentes que encontraron fueron dolor, sangrado y ulceración de la superficie cutánea.

Histológicamente la fascitis nodular puede clasificarse en tres subtipos, según el plano de tejido donde se origina: subcutáneo, intramuscular y fascial. Las lesiones normalmente nacen de la fascia y crecen extendiéndose a la grasa subcutánea en un crecimiento regular. Suelen ser bien delimitadas pero no encapsuladas. Las mitosis son abundantes, pero no se ven atipias celulares ${ }^{9}$. El subtipo subcutáneo se presenta de tres a diez veces más que los otros, y el tipo intramuscular es el que remeda con mayor frecuencia a una tumoración maligna, debido a su mayor tamaño y a su localización más profunda. El subtipo intramuscular es relativamente más frecuente en cabeza y cuello que en otras áreas anatómicas, es el peor circunscrito y crece a lo largo del plano fascial $^{1,2,9,12,14}$.

La patogénesis de la fascitis nodular continúa aún siendo desconocida. Bernstein et al postularon que la tumoración era una forma atípica de tejido de granulación quizás por un traumatismo menor previo, pero en las largas series de estudios publicados, sólo en un pequeño porcentaje de pacientes se ha podido demostrar tal asociación. También se ha relacionado una alteración en el cromosoma 3, en la región 3q21, con la presencia de fascitis nodular, aunque deberían realizarse más estudios para concluir que existe una predisposición genética para su aparición $n^{2,4,8,10,12,14}$.

En cuanto a su posible asociación con el período gestacional, el embarazo se considera una situación médica coexistente en ese tiempo. Sin embargo, en el estudio de Anand et $\mathrm{al}^{15}$ los autores intentaron relacionar las alteraciones hormonales propias del embarazo con la aparición de la lesión, y más concretamente con un aumento celular de receptores hormonales estrogénicos. En la fascitis nodular se ha demostrado la presencia de receptores estrogénicos en las células tumorales. Basándose en su caso, los autores proponen que el embarazo y los cambios estrogénicos relacionados con el mismo constituyen otro factor de riesgo para el desarrollo de la fascitis nodular, jugando un papel importante en la etiopa- togenia, ya que inducen cambios proliferativos en los miofibroblastos de las fascias.

A nivel de las pruebas de imagen, las características radiológicas de la fascitis nodular no son específicas. Tanto en la TC como en la RM, la fascitis nodular se observa como una lesión generalmente bien definida, homogénea, de los tejidos blandos, localizada a nivel superficial.

Aunque histológicamente es benigna, las lesiones profundas pueden mostrar un comportamiento clínico agresivo, simulando una tumoración maligna ${ }^{3,12,14}$. El diagnóstico diferencial incluye tumoraciones benignas tales como neurofibroma mixoide, schwanoma, tumor neurogénico, tumor de glándula salivar menor, tumor dermoide o epidermoide, hemangioma, sarcoidosis, dermatofibroma, histiocitoma fibroso y miofibroma, o lesiones malignas como el dermatofibrosarcoma mixoide protuberans, neurofibrosarcoma, fibrosarcoma y el histiocitoma fibroso maligno. El diagnóstico definitivo de la lesión lo proporciona el estudio anatomopatológico $5,10,11,14$.

Cuando el diagnóstico está establecido, el tratamiento de elección es la exéresis local simple, evidenciándose una tasa de recurrencia en torno al 7\%. La presencia de recurrencia de la lesión nos debe hacer dudar del diagnóstico de fascitis nodular, replanteando el caso y el estudio histológico previo para buscar la presencia de una tumoración maligna. La regresión espontánea de la lesión se ha observado ocasionalmente. La mejora en el diagnóstico previo de la fascitis nodular es esencial para evitar sobretratamientos y disminuir la morbilidad en el tratamiento de los pacientes $3,4,10-12,14$.

\section{Agradecimientos}

A mi esposa por su ayuda.

\section{B I B L I O G R A F Í A}

1. Chun-Ding H, Yun-Ho L, Fei-Peng L. Postauricular nodular fascitis. Otolaryngol Head Neck Surg. 2007;137:164-5.

2. Silva P, Bruce IA, Malik T, Homer J, Banerjee S. Nodular fasciitis of the head and neck. J Laringol Otol. 2005;119:8-11. 
3. Milo A, Vito K, Kinney S, Bauer T. Nodular fasciitis arising in the external auditory canal. Otolaryngol Head Neck Surg. 1999;120: 591-2.

4. Thompson L. Nodular fascitis. Ear Nose Throat J. 2002;81:830.

5. Saad R, Takei H, Lipscomb J, Ruiz B. Nodular fasciitis of parotid region: A pitfaal in the diagnosis of pleomorphic adenomas on fine-needle aspiration cytology. Diagn Cytopathol. 2005;33:191-4.

6. Pereira C, Edwards M. Parotid nodular fasciitis in a mobile phone user. J Laringol Otol. 2000;114:886-7.

7. Fischer J, Abdul-Karim F, Robinson R. Intraparotid nodular fasciitis. Arch Pathol Lab Med. 1989;113:1276-8.

8. Van Royen C, Wackens G, Goossens A. Nodular fasciitis in a temporomandibular joint. Int J Oral Maxillofac Surg. 1993;22: 168-70.

9. Han W, Hu Q Yang X, Wang Z, Huang X. Nodular fasciitis in the orofacial region. Int J Oral Maxillofac Surg. 2006;35:924-7.
10. Nair P, Barrett A, Theodossy T. Oral nodular fasciitis: case report. Br J Oral Maxillofac Surg. 2004;42:360-2.

11. Dahl I, Jarlstedt J. Nodular fasciitis in the head and neck. Acta Otolaryngol. 1980;90:152-9.

12. Shin JH, Lee HK, Cho KJ, Han MH, Na DG, Choi CG, et al. Nodular fasciitis of the head and neck radiographic findings. J Clin Imag. 2003;27:31-7.

13. Dayan D, Nasrallah V, Vered M. Clinico-pathologic correlations of myofibroblastic tumors of the oral cavity: I. Nodular fasciitis. J Oral Pathol Med. 2005;34:426-5.

14. Kim ST, Kim HJ, Park SW, Baek JH, Byun HS, Kim YM. Nodular fasciitis in the head and neck: CT and MR imaging findings. Am J Neuroradiol. 2005;26:2617-23.

15. Anand A, Tsapakis EM, Narvani AA, Alhakim A, Cannon S, Tsiridis E. Pseudosarcoma in a pregnant woman. World J Surg Oncol. 2007;18:5-7. 